\title{
017 PP PEOPLE WITH DEMENTIA DOING RESEARCH: THE CHALLENGES FOR PARTICIPATION AND COLLABORATION
}

\author{
L Birt, ${ }^{1 *} \mathrm{C}$ di Lorito, ${ }^{2}$ J Waite, ${ }^{3} \mathrm{P}$ Higgs, ${ }^{4} \mathrm{~F}$ Poland, ${ }^{1}$ P Leung, ${ }^{4}$ \\ $G$ Charlesworth' ${ }^{3}$ ' 'School of Health Sciences, University of East Anglia, UK; \\ ${ }^{2}$ Division of Psychiatry and Applied Psychology, University of Nottingham, UK; \\ ${ }^{3}$ Research Department of Clinical, Educational and Health Psychology, University \\ College London, UK.; ${ }^{4}$ Division of Psychiatry, University College London, UK
}

\subsection{6/bmjopen-2017-016492.35}

A diagnosis of dementia often shifts a person's social status. Opportunities for the person with dementia to be autonomous in decisions and agentic in constructing access to community activities can diminish. Reduced engagement with others may be attributed to cognitive impairment, but it may, even if unintentionally, be a consequence of the action of others.

Our aim is to explore the social structures which enable or restrict opportunities for the person with dementia to collaborate with researchers. We draw on empirical literature, researchers' reflections and participants' words. The data were collected during a qualitative study exploring the experiences and meaning of social participation for people living with mild dementia (The PRIDE study). We use participation in research as an exemplar of a situation where a diagnosis of dementia triggers a social positioning as 'vulnerable'; whereby competence to consent is questioned and family views may take precedence. The research procedures designed to protect people with dementia can have the consequence of limiting opportunities for their participation in research. Our research design is novel in that we are seeking to work with people with dementia as collaborators. Peer research, or co research, is a developing method in qualitative research and the intricacy of peer research with people with dementia is underexplored and theoretically unchallenged. We report on the processes of engaging people with dementia in doing research, highlighting examples of good practice. 